\title{
Convergence and Data Dependence Results for AK Iterative Algorithm
}

\author{
Müzeyyen Ertürk \\ Department of Mathematics, Art and Science Faculty, Adıyaman University, Adıyaman, Turkey, \\ merturk3263@gmail.com
}

Received: 8 February 2017

Accepted: 31 August 2017

DOI: $10.18466 /$ cbayarfbe.339352

\begin{abstract}
This paper concentrates on studying convergence and data dependence of AK iteration for the class of maps which was introduced by Berinde. Also, we will support our results with an example.

Keywords - Class of Maps, Convergence of Iteration, Data Dependency, Fixed Point, Iteration
\end{abstract}

\section{Introduction}

Fixed point theory has been built on the solutions of $T x=$ $x$. One of most famous theorems of this theory is Banach contraction theorem. This theorem states that if $T: X \rightarrow X$ provides contraction condition such that

$$
d(T x, T y) \leq k d(x, y)
$$

where $k \in(0,1]$, then it is a unique fixed point in the complete metric space. Successive approximations method called iteration which used in the proof of this theorem has inspired many researchers to approach the fixed point of maps classes with different properties. Finding the iterations that converge to the fixed point of a map has turned into a remarkably interesting field in the fixed point theory (see [614]).

On the other hand, it is not always possible to find fixed point of a map analytically. In such situations, fixed point of a approximate operator of $T$ can be regarded as the approximate solution of $T x=x$. In this context, estimating the distance between the fixed points of $T$ and its a approximate operator is an important problem. There exist many papers on this subject in the literature. Some of them can be listed as [15-19].

Let's give some preliminary informations to understand our purpose in this article.

Definition 1 [5] Let $(X, d)$ be a metric space. $T$ is called a Zamfirescu map if there exist $a_{1} \in(0,1), a_{2}, a_{3} \in$ $\left(0, \frac{1}{2}\right)$ such that at least one of the followings is true for each $x, y \in X$.

i) $\quad d(T x, T y) \leq a_{1} d(x, y)$

ii) $\quad d(T x, T y) \leq a_{2}[d(x, T x)+d(y, T y)]$

iii) $\quad(T x, T y) \leq a_{3}[d(x, T y)+d(y, T x)]$
In [5], it was shown that Picard iteration related with Zamfirescu map is convergent to a unique fixed point of $T$. Zamfirescu's theorem is very important since it generalizes Banach contraction theorem to non-continuous map. Then, in [1], Berinde proved that Ishikawa iteration for Zamfirescu map is convergent to its fixed point. In the proof, he showed that a Zamfirescu map can be written as the follows:

$$
d(T x, T y) \leq \delta d(x, y)+2 \delta d(x, T x)
$$

We can write (1.1) as (1.2) in the normed spaces:

$$
\|T x-T y\| \leq \delta\|x-y\|+2 \delta\|x-T x\| \text {. }
$$

If you pay attention, the class of maps satisfying (1.2) is more general than the class of contraction maps.

Recently, Ullah and Arshad [4] introduced the following new iteration process known as AK iteration process to approximate fixed point of contraction maps:

$$
\left\{\begin{array}{c}
x_{n+1}=T y_{n} \\
y_{n}=T\left(\left(1-\alpha_{n}\right) z_{n}+\alpha_{n} T z_{n}\right) \\
z_{n}=T\left(\left(1-\beta_{n}\right) x_{n}+\beta_{n} T x_{n}\right)
\end{array}\right.
$$

Our first target in this article is to approach the fixed point of the class of maps satisfying (1.2) by means of AK iteration method. The second is to estimate distance between the fixed points of a map satisfying (1.2) and its any approximate operator with the help of AK iteration. In addition, we will give an example that illustrate our results.

\section{Main Results}

In this section, we give the convergence and data results of 
iterative sequence (1.3) in the normed space. Furthermore, we will concretize our results with an examples. First, let's give the following lemma to shorten proofs of our theorems.

Lemma 1 Let $X$ be a normed space, $T: X \rightarrow X$ be an operator satisfying (1.2) with fixed point $x^{*}$. Then

$$
\left\|T x-x^{*}\right\| \leq \delta\left\|x-x^{*}\right\| .
$$

Proof It is easly seen that

$\left\|T x-x^{*}\right\| \leq \delta\left\|x-x^{*}\right\|+2 \delta\left\|x^{*}-T x^{*}\right\|=\delta\left\|x-x^{*}\right\|$.

Theorem 1 Let $T: X \rightarrow X$ be an operator satisfying (1.2) and $\left\{x_{n}\right\}_{n=0}^{\infty}$ be the iterative sequence (1.3) with real sequences $\left\{\alpha_{n}\right\}_{n=0}^{\infty}$ and $\left\{\beta_{n}\right\}_{n=0}^{\infty}$ in $[0,1]$ satisfying $\sum_{k=0}^{\infty} \beta_{k}=$ $\infty$. Then $\left\{x_{n}\right\}_{n=0}^{\infty}$ converges strongly to unique fixed point $x^{*}$ of $T$.

Proof Using (1.2), (1.3) and (2.1), we obtain that

$$
\begin{aligned}
& \left\|x_{n+1}-x^{*}\right\|=\left\|T y_{n}-x^{*}\right\| \leq \delta\left\|y_{n}-x^{*}\right\|, \\
& \left\|y_{n}-x^{*}\right\|=\left\|T\left(\left(1-\alpha_{n}\right) z_{n}+\alpha_{n} T z_{n}\right)-x^{*}\right\| \\
& \leq \delta\left\|\left(1-\alpha_{n}\right) z_{n}+\alpha_{n} T z_{n}-x^{*}\right\| \\
& \leq \delta\left(1-\alpha_{n}\right)\left\|z_{n}-x^{*}\right\| \\
& +\delta \alpha_{n}\left\|T z_{n}-x^{*}\right\| \\
& \leq \delta\left(1-\alpha_{n}\right)\left\|z_{n}-x^{*}\right\| \\
& +\delta^{2} \alpha_{n}\left\|z_{n}-x^{*}\right\| \\
& \leq \delta\left(1-\alpha_{n}(1-\delta)\right)\left\|z_{n}-x^{*}\right\| \text {, }
\end{aligned}
$$

And

$$
\begin{aligned}
\left\|z_{n}-x^{*}\right\|=\| T & \left.\left(1-\beta_{n}\right) x_{n}+\beta_{n} T x_{n}\right)-x^{*} \| \\
& \leq \delta\left\|\left(1-\beta_{n}\right) x_{n}+\beta_{n} T x_{n}-x^{*}\right\| \\
& \leq \delta\left(1-\beta_{n}\right)\left\|x_{n}-x^{*}\right\| \\
& +\delta \beta_{n}\left\|T x_{n}-x^{*}\right\| \\
& \leq \delta\left(1-\beta_{n}\right)\left\|x_{n}-x^{*}\right\| \\
& +\delta^{2} \beta_{n}\left\|x_{n}-x^{*}\right\| \\
& =\delta\left(1-\beta_{n}(1-\delta)\right)\left\|x_{n}-x^{*}\right\| .
\end{aligned}
$$

Substituting (2.4) and (2.3) in (2.2), we obtain

$$
\begin{aligned}
& \left\|x_{n+1}-x^{*}\right\| \\
& \leq \delta^{3}\left(1-\alpha_{n}(1-\delta)\right)\left(1-\beta_{n}(1-\delta)\right)\left\|x_{n}-x^{*}\right\| .
\end{aligned}
$$

By using the fact that $1-\alpha_{n}(1-\delta)<1$, we re-write (2.5) as follows:

$$
\left\|x_{n+1}-x^{*}\right\| \leq \delta^{3}\left(1-\beta_{n}(1-\delta)\right)\left\|x_{n}-x^{*}\right\| .
$$

Considering (2.6), we have

$$
\left\|x_{n+1}-x^{*}\right\| \leq \delta^{3}\left(1-\beta_{n}(1-\delta)\right)\left\|x_{n}-x^{*}\right\|
$$

$$
\leq \delta^{3}\left(1-\beta_{n}(1-\delta)\right) \delta^{3}\left(1-\beta_{n-1}(1-\delta)\right)\left\|x_{n-1}-x^{*}\right\|
$$

$\leq \cdots$

$\leq \delta^{3(n+1)} \prod_{k=0}^{n}\left[1-\beta_{k}(1-\delta)\right]\left\|x_{0}-x^{*}\right\|$.

It is well-known from the classical analysis that $1-x \leq$ $e^{-x}$ for all $x \in[0,1]$. Taking into account these facts together with (2.7), we obtain

$$
\left\|x_{n+1}-x^{*}\right\| \leq \delta^{3(n+1)} e^{-(1-\delta) \sum_{k=0}^{n} \beta_{k}}\left\|x_{0}-x^{*}\right\| .
$$

Taking the limit of both sides of inequality (2.8) yields $\lim _{n \rightarrow \infty}\left\|x_{n+1}-x^{*}\right\|=0$, i.e. $x_{n} \rightarrow x^{*}$ for $n \rightarrow \infty$, as required.

Now, we show that $x^{*}$ is unique fixed point of $T$. Assume that $x_{1}^{*}$ is another fixed point of $T$. If we write $x:=x_{1}^{*}$ in Lemma 1 , we obtain

$$
\left\|x_{1}^{*}-x^{*}\right\|=\left\|T x_{1}^{*}-x^{*}\right\| \leq \delta\left\|x_{1}^{*}-x^{*}\right\| .
$$

(2.9) is only possible in the case of $x^{*}=x_{1}^{*}$. This means that $x^{*}$ is unique fixed point of $T$.

Remark 1 Indeed, Theorem 1 is also true while only one of control sequences $\left\{\alpha_{n}\right\}_{n=0}^{\infty}$ and $\left\{\beta_{n}\right\}_{n=0}^{\infty}$ in iterative sequence (1.3) satisfies $\sum_{k=0}^{\infty} \alpha_{k}=\infty$ or $\sum_{k=0}^{\infty} \beta_{k}=\infty$.

Definition 2 [2] Let $T, \hat{T}: X \rightarrow X$ be operators. $\widehat{T}$ is called an approximate operator for $T$ if there exist some $\epsilon>0$ such that

$$
\|T x-\widehat{T} x\|<\epsilon, \forall x \in X .
$$

Lemma 2 [3] Let $\left\{\mu_{n}\right\}_{n=0}^{\infty},\left\{v_{n}\right\}_{n=0}^{\infty}$ and $\left\{\xi_{n}\right\}_{n=0}^{\infty}$ be nonnegative real sequences with $v_{n} \in(0,1)$ for all $n \in$ $\mathbb{N}, \sum_{k=0}^{\infty} v_{n}=\infty$. Suppose there exists $n_{0} \in \mathbb{N}$ such that for all $n \geq n_{0}$ one has the inequality

$$
\mu_{n+1} \leq\left(1-v_{n}\right) \mu_{n}+v_{n} \xi_{n} .
$$

Then the following inequality holds:

$0 \leq \limsup _{n \rightarrow \infty} \mu_{n} \leq \limsup _{n \rightarrow \infty} \xi_{n}$.

Theorem 2 Let $T: \mathrm{X} \rightarrow \mathrm{X}$ be an operator satisfying (1.2) with the fixed point $x^{*}, \widehat{T}: X \rightarrow X$ be an approximate operator of $T$ for given $\epsilon$, and $\hat{x}$ be the fixed points of $\hat{T}$. Assume that $\left\{x_{n}\right\}_{n=0}^{\infty}$ is the sequence in (1.3) and $\left\{\hat{x}_{n}\right\}_{n=0}^{\infty}$ is a sequence defined by

$$
\left\{\begin{array}{c}
\hat{x}_{n+1}=\hat{T} \hat{y}_{n} \\
\hat{y}_{n}=\hat{T}\left(\left(1-\alpha_{n}\right) \hat{z}_{n}+\alpha_{n} \hat{T} \hat{z}_{n}\right) \\
\hat{z}_{n}=\hat{T}\left(\left(1-\beta_{n}\right) \hat{x}_{n}+\beta_{n} \hat{T} \hat{x}_{n}\right)
\end{array}\right.
$$


where $\left\{\alpha_{n}\right\}_{n=0}^{\infty}$ and $\left\{\beta_{n}\right\}_{n=0}^{\infty}$ are real sequences in $[0,1]$ satisfying $\lim _{n \rightarrow \infty} \alpha_{n}=\lim _{n \rightarrow \infty} \beta_{n}=0$ and $\sum_{k=0}^{\infty} \alpha_{k}=\infty$ or $\sum_{k=0}^{\infty} \beta_{k}=\infty$. If $\left\{x_{n}\right\}_{n=0}^{\infty} \infty \underset{n}{n \rightarrow \infty}$ converges to $\hat{x}$, then we have

$$
\left\|x^{*}-\hat{x}\right\| \leq \frac{\epsilon}{1-\delta} .
$$

Proof Using (1.2), (1.3), (2.1) and (2.10), we get

$$
\begin{aligned}
\left\|x_{n+1}-\hat{x}_{n+1}\right\|= & \| T y_{n} \\
& -\hat{T} \hat{y}_{n} \| \\
& \leq\left\|T y_{n}-T \hat{y}_{n}\right\|+\left\|T \hat{y}_{n}-\hat{T} \hat{y}_{n}\right\| \\
& \leq \delta\left\|y_{n}-\hat{y}_{n}\right\|+2 \delta\left\|T y_{n}-y_{n}\right\|+\epsilon \\
& \leq \delta\left\|y_{n}-\hat{y}_{n}\right\|+2 \delta\left\|T y_{n}-x^{*}\right\| \\
& +2 \delta\left\|x^{*}-y_{n}\right\|+\epsilon \\
& \leq \delta\left\|y_{n}-\hat{y}_{n}\right\|+2 \delta^{2}\left\|y_{n}-x^{*}\right\| \\
& +2 \delta\left\|x^{*}-y_{n}\right\|+\epsilon \\
& =\delta\left\|y_{n}-\hat{y}_{n}\right\|+\left(2 \delta^{2}+2 \delta\right)\left\|y_{n}-x^{*}\right\|
\end{aligned}
$$

and

$$
\begin{aligned}
& \left\|y_{n}-\hat{y}_{n}\right\|=\left\|\begin{array}{c}
T\left(\left(1-\alpha_{n}\right) z_{n}+\alpha_{n} T z_{n}\right) \\
-\widehat{T}\left(\left(1-\alpha_{n}\right) \hat{z}_{n}+\alpha_{n} \hat{T}_{\hat{z}}\right)
\end{array}\right\| \\
& \leq\left\|\begin{array}{c}
T\left(\left(1-\alpha_{n}\right) z_{n}+\alpha_{n} T z_{n}\right) \\
-T\left(\left(1-\alpha_{n}\right) \hat{z}_{n}+\alpha_{n} \hat{T} \hat{z}_{n}\right)
\end{array}\right\| \\
& +\left\|\begin{array}{c}
T\left(\left(1-\alpha_{n}\right) \hat{z}_{n}+\alpha_{n} \hat{T} \hat{z}_{n}\right) \\
-\widehat{T}\left(\left(1-\alpha_{n}\right) \hat{z}_{n}+\alpha_{n} \hat{T} \hat{z}_{n}\right)
\end{array}\right\| \\
& \leq \delta\left\|\begin{array}{c}
\left(1-\alpha_{n}\right) z_{n}+\alpha_{n} T z_{n} \\
-\left(1-\alpha_{n}\right) \hat{z}_{n}-\alpha_{n} \hat{T} \hat{z}_{n}
\end{array}\right\| \\
& +2 \delta\left\|\begin{array}{c}
T\left(\left(1-\alpha_{n}\right) z_{n}+\alpha_{n} T z_{n}\right) \\
-\left(1-\alpha_{n}\right) z_{n}-\alpha_{n} T z_{n}
\end{array}\right\|+\epsilon \\
& \leq \delta\left[\left(1-\alpha_{n}\right)\left\|z_{n}-\hat{z}_{n}\right\|+\alpha_{n}\left\|T z_{n}-\hat{T} \hat{z}_{n}\right\|\right] \\
& +2 \delta\left[\begin{array}{c}
\left\|T\left(\left(1-\alpha_{n}\right) z_{n}+\alpha_{n} T z_{n}\right)-x^{*}\right\| \\
+\left\|x^{*}-\left(1-\alpha_{n}\right) z_{n}-\alpha_{n} T z_{n}\right\|
\end{array}\right] \\
& +\epsilon \\
& \leq \delta\left[\begin{array}{c}
\left(1-\alpha_{n}\right)\left\|z_{n}-\hat{z}_{n}\right\| \\
+\alpha_{n}\left\|T z_{n}-T \hat{z}_{n}\right\|+\alpha_{n}\left\|T \hat{z}_{n}-\hat{T} \hat{z}_{n}\right\|
\end{array}\right] \\
& +2 \delta\left[\begin{array}{l}
\delta\left\|\left(1-\alpha_{n}\right) z_{n}+\alpha_{n} T z_{n}-x^{*}\right\| \\
+\left\|x^{*}-\left(1-\alpha_{n}\right) z_{n}-\alpha_{n} T z_{n}\right\|
\end{array}\right] \\
& +\epsilon \\
& \leq \delta\left[\begin{array}{c}
\left(1-\alpha_{n}\right)\left\|z_{n}-\hat{z}_{n}\right\| \\
+\alpha_{n}\left\|T z_{n}-T \hat{z}_{n}\right\|+\alpha_{n}\left\|T \hat{z}_{n}-\hat{T} \hat{z}_{n}\right\|
\end{array}\right] \\
& +2 \delta(\delta+1) \| x^{*}-\left(1-\alpha_{n}\right) z_{n} \\
& -\alpha_{n} T z_{n} \|+\epsilon \\
& \leq \delta\left[\begin{array}{c}
\left(1-\alpha_{n}\right)\left\|z_{n}-\hat{z}_{n}\right\| \\
+\alpha_{n}\left(\delta\left\|z_{n}-\hat{z}_{n}\right\|+2 \delta\left\|T z_{n}-z_{n}\right\|\right)+\alpha_{n} \epsilon
\end{array}\right] \\
& +2 \delta(\delta+1)\left[\left(1-\alpha_{n}\right)\left\|z_{n}-x^{*}\right\|\right. \\
& \left.+\alpha_{n}\left\|x^{*}-T z_{n}\right\|\right]+\epsilon
\end{aligned}
$$

and

$$
\begin{gathered}
\leq \delta\left[\begin{array}{c}
\left(1-\alpha_{n}\right)\left\|z_{n}-\hat{z}_{n}\right\|+\alpha_{n} \delta\left\|z_{n}-\hat{z}_{n}\right\| \\
\left.+2 \alpha_{n} \delta\left\|T z_{n}-x^{*}\right\|+2 \alpha_{n} \delta\left\|x^{*}-z_{n}\right\|\right)+\alpha_{n} \epsilon
\end{array}\right] \\
+2 \delta(\delta+1)\left[\left(1-\alpha_{n}\right)\left\|z_{n}-x^{*}\right\|\right. \\
\left.+\alpha_{n} \delta\left\|z_{n}-x^{*}\right\|\right]+\epsilon \\
\leq \delta\left[\begin{array}{c}
\left(1-\alpha_{n}\right)\left\|z_{n}-\hat{z}_{n}\right\|+\alpha_{n} \delta\left\|z_{n}-\hat{z}_{n}\right\| \\
+2 \alpha_{n} \delta^{2}\left\|z_{n}-x^{*}\right\|+2 \alpha_{n} \delta\left\|z_{n}-x^{*}\right\|+\alpha_{n} \epsilon
\end{array}\right] \\
+2 \delta(\delta+1)\left[\left(1-\alpha_{n}\right)+\alpha_{n} \delta\right] \| z_{n} \\
-x^{*} \|+\epsilon \\
\leq \delta\left(1-\alpha_{n}(1-\delta)\left\|z_{n}-\hat{z}_{n}\right\|\right. \\
+\left[\begin{array}{c}
2 \delta^{2} \alpha_{n}+2 \delta \alpha_{n} \\
+2 \delta(\delta+1)\left(1-\alpha_{n}\right)+2 \delta(\delta+1) \alpha_{n} \delta
\end{array}\right]\left\|z_{n}-x^{*}\right\| \\
+\alpha_{n} \epsilon \delta+\epsilon \quad \\
+\delta\left(1-\alpha_{n}(1-\delta)\left\|z_{n}-\hat{z}_{n}\right\|\right. \\
+\left[\begin{array}{c}
2 \delta^{2} \alpha_{n}+2 \delta \alpha_{n}+\left(2 \delta^{2}+2 \delta\right) \\
-2 \delta^{2} \alpha_{n}-2 \delta \alpha_{n}+2 \delta(\delta+1) \alpha_{n} \delta
\end{array}\right]\left\|z_{n}-x^{*}\right\|+\alpha_{n} \epsilon \delta \\
+\epsilon \quad \begin{array}{c} 
\\
=\delta\left(1-\alpha_{n}(1-\delta)\left\|z_{n}-\hat{z}{ }_{n}\right\|\right. \\
+2 \delta(\delta+1)\left(1+\alpha_{n} \delta\right)\left\|z_{n}-x^{*}\right\| \\
+\alpha_{n} \epsilon \delta+\epsilon
\end{array}
\end{gathered}
$$$$
+\epsilon
$$

$$
\begin{aligned}
& \left\|z_{n}-\hat{z}_{n}\right\|=\left\|\begin{array}{c}
T\left(\left(1-\beta_{n}\right) x_{n}+\beta_{n} T x_{n}\right) \\
-\hat{T}\left(\left(1-\beta_{n}\right) \hat{x}_{n}+\beta_{n} \hat{T} \hat{x}_{n}\right)
\end{array}\right\| \\
& \leq\left\|\begin{array}{c}
T\left(\left(1-\beta_{n}\right) x_{n}+\beta_{n} T x_{n}\right) \\
-T\left(\left(1-\beta_{n}\right) \hat{x}_{n}+\beta_{n} \hat{T} \hat{x}_{n}\right)
\end{array}\right\| \\
& +\left\|\begin{array}{c}
T\left(\left(1-\beta_{n}\right) \hat{x}_{n}+\beta_{n} \hat{T} \hat{x}_{n}\right) \\
-\widehat{T}\left(\left(1-\beta_{n}\right) \hat{x}_{n}+\beta_{n} \hat{T} \hat{x}_{n}\right)
\end{array}\right\| \\
& \leq \delta\left\|\begin{array}{c}
\left(1-\beta_{n}\right) x_{n}+\beta_{n} T x_{n} \\
-\left(1-\beta_{n}\right) \hat{x}_{n}-\beta_{n} \hat{T} \hat{x}_{n}
\end{array}\right\| \\
& +2 \delta\left\|\begin{array}{c}
T\left(\left(1-\beta_{n}\right) x_{n}+\beta_{n} T x_{n}\right) \\
-\left(1-\beta_{n}\right) x_{n}-\beta_{n} T x_{n}
\end{array}\right\|+\epsilon \\
& \leq \delta\left[\begin{array}{c}
\left(1-\beta_{n}\right)\left\|x_{n}-\hat{x}_{n}\right\| \\
+\beta_{n}\left\|T x_{n}-\hat{T} \hat{x}_{n}\right\|
\end{array}\right] \\
& +2 \delta\left[\begin{array}{c}
\left\|T\left(\left(1-\beta_{n}\right) x_{n}+\beta_{n} T x_{n}\right)-x^{*}\right\| \\
+\left\|x^{*}-\left(1-\beta_{n}\right) x_{n}-\beta_{n} T x_{n}\right\|
\end{array}\right]+\epsilon \\
& \leq \delta\left[\begin{array}{c}
\left(1-\beta_{n}\right)\left\|x_{n}-\hat{x}_{n}\right\| \\
+\beta_{n}\left\|T x_{n}-T \hat{x}_{n}\right\|+\beta_{n}\left\|T \hat{x}_{n}-\hat{T} \hat{x}_{n}\right\|
\end{array}\right] \\
& +2 \delta\left[\begin{array}{l}
\delta\left\|\left(1-\beta_{n}\right) x_{n}+\beta_{n} T x_{n}-x^{*}\right\| \\
+\left\|x^{*}-\left(1-\beta_{n}\right) x_{n}+\beta_{n} T x_{n}\right\|
\end{array}\right] \\
& +\epsilon \\
& \leq \delta\left[\begin{array}{c}
\left(1-\beta_{n}\right)\left\|x_{n}-\hat{x}_{n}\right\| \\
+\beta_{n}\left(\delta\left\|x_{n}-\hat{x}_{n}\right\|+2 \delta\left\|x_{n}-T x_{n}\right\|\right)+\beta_{n} \epsilon
\end{array}\right] \\
& +2 \delta(\delta+1) \| x^{*}-\left(1-\beta_{n}\right) x_{n} \\
& -\beta_{n} T x_{n} \|+\epsilon \\
& \leq \delta\left[\begin{array}{c}
\left(1-\beta_{n}\right)\left\|x_{n}-\hat{x}_{n}\right\|+\beta_{n} \delta\left\|x_{n}-\hat{x}_{n}\right\| \\
+2 \beta_{n} \delta\left\|x^{*}-T x_{n}\right\|+2 \beta_{n} \delta\left\|x_{n}-x^{*}\right\|+\beta_{n} \epsilon
\end{array}\right] \\
& +2 \delta(\delta+1)\left[\begin{array}{c}
\left(1-\beta_{n}\right)\left\|x_{n}-x^{*}\right\| \\
+\beta_{n} \delta\left\|x_{n}-x^{*}\right\|
\end{array}\right]+\epsilon
\end{aligned}
$$


Celal Bayar University Journal of Science

Volume 13, Issue 3, p 755-759

M. Ertürk

$$
\begin{gathered}
\leq \delta\left[\begin{array}{c}
\left(1-\beta_{n}\right)\left\|x_{n}-\hat{x}_{n}\right\|+\beta_{n} \delta\left\|x_{n}-\hat{x}_{n}\right\| \\
+2 \beta_{n} \delta^{2}\left\|x^{*}-x_{n}\right\|+2 \beta_{n} \delta\left\|x_{n}-x^{*}\right\|+\beta_{n} \epsilon
\end{array}\right] \\
+2 \delta(\delta+1)\left[\begin{array}{c}
\left(1-\beta_{n}\right)\left\|x_{n}-x^{*}\right\| \\
+\beta_{n} \delta\left\|x_{n}-x^{*}\right\|
\end{array}\right]+\epsilon \\
\leq\left[\delta\left(1-\beta_{n}\right)+\beta_{n} \delta^{2}\right]\left\|x_{n}-\hat{x}_{n}\right\| \\
+\left[\begin{array}{c}
2 \beta_{n} \delta^{3}+2 \beta_{n} \delta^{2} \\
+2 \delta(\delta+1)\left(1-\beta_{n}\right) \\
+2 \delta(\delta+1) \beta_{n} \delta
\end{array}\right]\left\|x_{n}-x^{*}\right\| \\
+\beta_{n} \epsilon \delta+\epsilon
\end{gathered}
$$

$\leq \delta\left(1-\beta_{n}(1-\delta)\right)\left\|x_{n}-\hat{x}_{n}\right\|$

$+\left[\begin{array}{c}2 \delta^{2} \beta_{n}+2 \delta \beta_{n}+\left(2 \delta^{2}+2 \delta\right) \\ -2 \delta^{2} \beta_{n}-2 \delta \beta_{n}+2 \delta(\delta+1) \beta_{n} \delta\end{array}\right]\left\|x_{n}-x^{*}\right\|+\beta_{n} \epsilon \delta$

$+\epsilon$

$$
\begin{aligned}
=\delta\left(1-\beta_{n}(1-\right. & \delta))\left\|x_{n}-\hat{x}_{n}\right\| \\
& +2 \delta(\delta+1)\left(1+\beta_{n} \delta\right)\left\|x_{n}-x^{*}\right\| \\
& +\beta_{n} \epsilon \delta+\epsilon
\end{aligned}
$$

Substituting (2.14) in (2.13), we have

$\left\|y_{n}-\hat{y}_{n}\right\|$

$\leq \delta\left(\begin{array}{c}1 \\ -\alpha_{n}(1-\delta)\end{array}\right)\left(\begin{array}{c}\delta\left(1-\beta_{n}(1-\delta)\right)\left\|x_{n}-\hat{x}_{n}\right\| \\ +2 \delta(\delta+1)\left(1+\beta_{n} \delta\right)\left\|x_{n}-x^{*}\right\| \\ +\beta_{n} \epsilon \delta+\epsilon\end{array}\right)$

$+2 \delta(\delta+1)\left(1+\alpha_{n} \delta\right)\left\|z_{n}-x^{*}\right\|+\alpha_{n} \epsilon \delta+\epsilon$

Now, substitute (2.15) in (2.12), we get

$$
\begin{aligned}
& \| \delta\left(\begin{array}{c}
\delta x_{n+1}-\hat{x}_{n+1} \| \\
\delta\left(1-\alpha_{n}(1-\delta)\right)\left(\begin{array}{c}
\delta\left(1-\beta_{n}(1-\delta)\left\|x_{n}-\hat{x}_{n}\right\|\right. \\
+2 \delta(\delta+1)\left(1+\beta_{n} \delta\right)\left\|x_{n}-x^{*}\right\| \\
+\beta_{n} \epsilon \delta+\epsilon
\end{array}\right) \\
+2 \delta(\delta+1)\left(1+\alpha_{n} \delta\right)\left\|z_{n}-x^{*}\right\|+\alpha_{n} \epsilon \delta+\epsilon
\end{array}\right) \\
& +\left(2 \delta^{2}+2 \delta\right)\left\|y_{n}-x^{*}\right\|+\epsilon
\end{aligned}
$$

Since $\delta^{3} \in(0,1)$, there exists a real number $\rho \in(0,1)$ such that

$$
\delta^{3}=1-\rho .
$$

Regarding (2.17) and using the facts of $1-\alpha_{n}(1-\delta)<1$ and $1-\beta_{n}(1-\delta)<1$, we can re-write (2.16) as $\left\|x_{n+1}-\hat{x}_{n+1}\right\|$

$\leq(1-\rho)\left\|x_{n}-\hat{x}_{n}\right\|$

$$
+\rho \frac{\left(\begin{array}{c}
2 \delta^{3}(\delta+1)\left(1+\beta_{n} \delta\right)\left\|x_{n}-x^{*}\right\| \\
+\left(2 \delta^{2}+2 \delta\right)\left\|y_{n}-x^{*}\right\| \\
+2 \delta^{2}(1+\delta)\left(1+\alpha_{n} \delta\right)\left\|z_{n}-x^{*}\right\| \\
+\alpha_{n} \epsilon \delta^{2}+\epsilon \delta+\beta_{n} \epsilon \delta^{3}+\epsilon \delta^{2}+\epsilon
\end{array}\right)}{\rho} .
$$

Denote

$$
\begin{aligned}
& \mu_{n}=\left\|x_{n+1}-\hat{x}_{n+1}\right\|, \\
& v_{n}=\rho, \\
& \xi_{n}=\left(\begin{array}{c}
2 \delta^{3}(\delta+1)\left(1+\beta_{n} \delta\right)\left\|x_{n}-x^{*}\right\| \\
+\left(2 \delta^{2}+2 \delta\right)\left\|y_{n}-x^{*}\right\| \\
+2 \delta^{2}(1+\delta)\left(1+\alpha_{n} \delta\right)\left\|z_{n}-x^{*}\right\| \\
+\alpha_{n} \epsilon \delta^{2}+\epsilon \delta+\beta_{n} \epsilon \delta^{3}+\epsilon \delta^{2}+\epsilon
\end{array}\right) .
\end{aligned}
$$

It is now easy to check that (2.18) provide the requirements in Lemma 2 and so, we have

$$
\left\|x^{*}-\hat{x}\right\| \leq \frac{\epsilon}{1-\delta} .
$$

Example 1 Let $X=[0,1]$ and consider abso absolute value norm on $[0,1]$. Let $T: X \rightarrow X$ be the map defined by

$$
T x=\left\{\begin{array}{rr}
-0.5+e^{-x}+\frac{\sin x^{2}}{2}, & x \in\left[0, \frac{1}{2}\right) \\
\frac{\cos x^{3}}{7}+\frac{x^{2}}{6}, & x \in\left[\frac{1}{2}, 1\right]
\end{array}\right.
$$

Fixed point of $T$ is $x^{*}=0.2901809$. $T$ satisfies condition (1.2) with $\delta=0.69509$. So, from Theorem 2, we say that (1.3) iterative sequence is convergent to $X^{*}=0.2901809$. Now, define $\widehat{T}: X \rightarrow X$ by

$\hat{T} x$

$$
=\left\{\begin{array}{cc}
\left(\begin{array}{cc}
0.25+0.056656 e^{\sin x}+0.002351 \sqrt{x} \\
-\frac{3.97105 x^{3}}{10^{5}}+\frac{3.18243 \cosh x}{10^{21716}}
\end{array}\right), & x \in\left[0, \frac{1}{2}\right) \\
\left(\begin{array}{cc}
-0.13+0.68197 e^{-x}+\frac{7.30137 x^{\frac{5}{2}}}{10^{13}} \\
-\frac{3.21895 \sinh (1.5708 x)}{10^{34111}}
\end{array}\right), & x \in\left[\frac{1}{2}, 1\right]
\end{array}\right.
$$

By using Wolfram Mathematica 9 software package, we get

$$
\|T x-\widehat{T} x\|<0.193344
$$

for all $x \in X$. This means that is $\widehat{T}$ is an approximate operator of $T$ for a fixed $\epsilon=0.193344$. On the other hand, fixed point of $\widehat{T}$ is $\hat{x}=0.329663$. Indeed, if we put $\alpha_{n}=\frac{1}{n+10}$, $\beta_{n}=\frac{1}{n^{2}+3}$ for all $n \in \mathbb{N}$ in (2.11), then we have

$$
\left\{\begin{array}{c}
\hat{x}_{n+1}=\hat{T} \hat{y}_{n}, \\
\hat{y}_{n}=\hat{T}\left(\left(1-\frac{1}{n+10}\right) \hat{z}_{n}+\frac{1}{n+10} \hat{T} \hat{z}_{n}\right), \\
\hat{z}_{n}\left(\left(1-\frac{1}{n^{2}+3}\right) \hat{x}_{n}+\frac{1}{n^{2}+3} \hat{T} \hat{x}_{n}\right)
\end{array}\right.
$$

The following table shows that the sequence $\left\{\hat{x}_{n}\right\}_{n=0}^{\infty}$ generated by (2.18) converges to the fixed point $\hat{x}=$ $0.329663 \in X$. 


\begin{tabular}{|c|c|}
\hline Number of Iterations & Iterative method (2.18) \\
\hline 0 & 0.4 \\
\hline 1 & 0.329834 \\
\hline 2 & 0.329663 \\
\hline 3 & 0.329663 \\
\hline 4 & 0.329663 \\
\hline$\vdots$ & $\vdots$ \\
\hline$\vdots$ & $\vdots$ \\
\hline
\end{tabular}

Hence, we obtain

$\left\|x^{*}-\hat{x}\right\|=0.0394821$.

Actually, without knowing and computing the fixed point $\hat{\mathrm{x}}$, we find the following estimate via Theorem 2, $\left\|x^{*}-\hat{x}\right\| \leq \frac{\epsilon}{1-\delta}=0.63410186$.

\section{References}

1. Berinde, V.; On the convergence of the Ishikawa iteration in the class of quasi contractive operators. Acta Mathematica Universitatis Comenianae, 2004, 73(1), 119-126.

2. Berinde, V.; Iterative approximation of fixed points; Springer: Berlin, Germany, 2007.

3. Schu, J.; Weak and strong convergence to fixed points of asymptotically nonexpansive mappings. Bulletin of the Australian Mathematical Society, 1991, 43(1), 153-159.

4. Ullah, K., and Arshad, M.; On diaerent results for new three step iteration process in Banach spaces. SpringerPlus 2016, 5(1), 1616.

5. Zamfirescu, T.; Fix point theorems in metric spaces. Archiv der Mathematik 1972, 23(1), 292-298.

6. Berinde, V.; General constructive fixed point theorems for Ciric-type almost contractions in metric spaces. Carpathian Journal of Mathematics. 2008, 24(2), 10-19.

7. Berinde, V., and Pacurar M.; Fixed points and continuity of almost contractions. Fixed Point Theory, 2008, 9(1), 23-34.
8. Karakaya, V., Doğan, K., Gürsoy, F., and Ertürk, M.; Fixed point of a new three-step iteration algorithm under contractivelike operators over normed spaces. Abstract and Applied Analysis, 2013, 2013, 1-9.

9. Khan, A. R., Gürsoy, F., and Karakaya, V.; Jungck-Khan iterative scheme and higher convergence rate. International Journal of Computer Mathematics 2016, 93(12), 1029-2105.

10. Gürsoy, F., Karakaya, V., and Rhoades, B. E.; Some convergence and stability results for the Kirk multistep and Kirk-SP fixed point iterative algorithms. Abstract and Applied Analysis, 2014, 2014, 1-12.

11. Xu, B., and Noor, M. A.; Fixed-point iterations for asymptotically nonexpansive mappings in Banach spaces. Journal of Mathematical Analysis and Applications, 2002, 67(2), 444-453.

12. Weng, X.; Fixed point iteration for local strictly pseudo-contractive mapping. Proceedings of the American Mathematical Society, 1991, 113(3), 727-731.

13. Rhoades, B. E.; Comments on two fixed point iteration methods. Journal of Mathematical Analysis and Applications 1976, 56(3), 741-750.

14. Tan, K.K., and $\mathrm{Xu}$, H.; Fixed point iteration processes for asymptotically nonexpansive mappings. Proceedings of the American Mathematical Society, 1994, 122(3), 733-739.

15. Soltuz, S. M., and Grosan, T.; Data dependence for Ishikawa iteration when dealing with contractive-like operators. Fixed Point Theory and Applications, 2008, 1, 1-7.

16. Şoltuz, Ş. M.; Data dependence for Mann iteration. Octogon Mathematical Magazine 2001, 9(2), 825-828.

17. Gürsoy, F., Karakaya, V., and Rhoades, B E.; Data dependence results of new multistep and S-iterative schemes for contractive-like operators. Fixed Point Theory and Applications, 2013, 76, 1-12.

18. Şahin, A., and Başarır M.; Convergence and data dependence results of an iteration process in a hyperbolic space. Filomat, 2016, 30(3), 569582.

19. Khan, A. R., Gürsoy, F., and Kumar V.; Stability and data dependence results for the Jungck-Khan iterative scheme. Turkish Journal of Mathematics 2016, 40, 631-640. 